\title{
Application of the Zürich Biofilm Model to Problems of Cariology
}

\author{
Bernhard Guggenheim ${ }^{a}$ Merlin Guggenheim ${ }^{a}$ Rudolf Gmüra \\ Elin Giertsen ${ }^{b}$ Thomas Thurnheer ${ }^{a}$ \\ a Institute for Oral Biology, Section for Oral Microbiology and General Immunology, University of Zürich, Zürich, \\ Switzerland; b Department of Odontology-Cariology, University of Bergen, Bergen, Norway
}

\section{Key Words}

Biofilms · Caries - Antimicrobials - Mouth rinses •

Demineralization $\cdot$ Remineralization $\cdot$ Three-dimensional morphology $\cdot$ Tortuosity

\begin{abstract}
The term biofilm is increasingly replacing 'plaque' in the literature, but concepts and existing paradigms are changing much more slowly. There is little doubt that biofilm research will lead to more realistic perception and interpretation of the physiology and pathogenicity of microorganisms colonizing plaques in the oral cavity. There is clear evidence that the genotypic and phenotypic expression profiles of biofilm and planktonic bacteria are different. Several techniques are available today to study multispecies biofilms of oral bacteria, each having its particular advantages and weaknesses. We describe a biofilm model developed in Zürich and demonstrate a number of applications with direct or indirect impact on prophylactic dentistry: spatial arrangement and associative behavior of various species in biofilms; multiplex fluorescent in situ hybridization analysis of oral bacteria in biofilms; use of the biofilm model to predict in vivo efficacy of antimicrobials reliably; mass transport in bio-
\end{abstract}

\section{KARGER}

Fax +41613061234

E-Mail karger@karger.ch

www. karger.com
(C) 2004 S. Karger AG, Basel

0008-6568/04/0383-0212\$21.00/0

Accessible online at:

www. karger.com/cre films; de- and remineralization of enamel exposed to biofilms in vitro. The potential of biofilm experimentation in oral biology has certainly not yet been fully exploited and dozens of possible interesting applications could be investigated. The overall physiological parameters of multispecies biofilms can be measured quite accurately, but it is still impossible to assess in toto the multitude of interactions taking place in such complex systems. What can and should be done is to test hypotheses stemming from experiments with planktonic cells in monospecies cultures. In particular, it will be interesting to investigate the relevance to biofilm composition and metabolism of specific gene products by using appropriate bacterial mutants.

Copyright $@ 2004$ S. Karger AG, Basel

Microorganisms colonizing the oral cavity had been a major research topic even before the epoch-making contributions of W.D. Miller who coined the prevailing view of the etiology of dental caries for decades. Acid formation from dietary starch by salivary bacteria, in particular by lactobacilli, emerged as a sustained paradigm to explain caries [Miller, 1973]. Early observations by Black and many others, who had pointed out the significance of
B. Guggenheim

Institute for Oral Biology, Section for Oral Microbiology and General Immunology

University of Zürich, Plattenstrasse 11

CH-8028 Zürich (Switzerland)

Tel. +41 44634 3277, Fax +41 44634 4310, E-Mail guggenhe@zzmk.unizh.ch 
the gelatinous nature of dental plaques and also the role of sucrose in the formation of these bacterial biofilms, were rapidly forgotten [Guggenheim, 1970]. The original version of the chemoparasitic caries theory of Miller received its 'coup de grâce' by Ron Gibbons in the early 1960s when it was shown that bacteria associated with dental caries must colonize supragingival plaque in high numbers [Gibbons, 1964]. This shifted mainstream research to plaque streptococci and in particular to mutans streptococci [Carlsson, 1967; Guggenheim, 1968]. Paul Keyes introduced the multifactorial caries theory [Keyes, 1960]. Animal experiments and both human cross-sectional and longitudinal studies left no doubt that mutans streptococci were strongly associated with enamel caries [Guggenheim et al., 1965; Hardie et al., 1977]. As a result, a most simple concept evolved: the specific plaque hypothesis. Dental caries was conceived as a mono-infection by mutans streptococci [Kristoffersson et al., 1985; Loesche, 1986]. The hypothesis induced a flood of in vitro studies with planktonically grown Streptococcus mutans and $S$. sobrinus cells, with the aim to pinpoint and elucidate virulence mechanisms of these streptococci [van Houte, 1994]. From these experiments, in part carried out with most modern molecular biological methods, a number of new paradigms emerged. Here, just a few will be mentioned. Primary bacterial adhesion to pellicle-coated enamel was described as a highly specific process, with the expression and nature of adhesins being the major determinants for the colonization of specific oral microhabitats [Liljemark and Bloomquist, 1996]. By analogy, secondary colonization was envisioned as entirely dependent on specific coadherence. It was considered predictable from in vitro tests assessing the coaggregation patterns of planktonic bacterial cells that had been mixed in suspensions under no-growth conditions [Kolenbrander and London, 1993]. Glucosyltransferases (GtfB, GtfC, GtfD) were claimed to play a crucial role in bacterial attachment, and their products were thought to act as diffusion-limiting macromolecules in plaque [Ooshima et al., 2001].

Only more recently has the picture started to shift. Studies with bacteria growing in biofilms, in particular in multispecies biofilms, clearly indicated that genotypic and phenotypic expression profiles of biofilm bacteria are different from those of planktonic bacteria [Costerton et al., 1994]. Such biofilm ecologies have been compared to higher multicellular organisms [Costerton et al., 1995]. They show complex intercellular interactions including communication by specific signaling molecules [Davies et al., 1998]. The resistance of biofilm bacteria against antimicrobials is increased [Gilbert et al., 1997]. Both the species and numerical composition of biofilms are dependent on the prevailing growth conditions. These seem ultimately decisive for the interaction with the host, resulting in health or disease. P.D. Marsh, a pioneer in oral biofilm experimentation, has described this relation as the 'ecological plaque hypothesis' [Marsh, 1994].

Various multispecies models of dental plaque have been described and applied to problems of clinical relevance, most notably biofilm permeability and chemical control of plaque. These systems usually consist either of flow cells [Christersson et al., 1987; Larsen and Fiehn, 1995; Sjollema et al., 1989] or of chemostats modified to allow for insertion and removal of colonizable surfaces [Bowden, 1999; Bradshaw et al., 1996; Herles et al., 1994; Kinniment et al., 1996]. While these devices have contributed to our understanding of microbial adhesion and biofilm formation, their use has certain drawbacks. They can be cumbersome to construct and/or difficult to maintain over long periods of time. Since clearance of pulsed substances is a function of flow rate and volume, chemostats operating with low flow rates and relatively large volumes can have quite long mean residence times, rendering them impractical for studies of selected compounds with shortterm exposures, as is common in oral hygiene procedures. Moreover, systems with working volumes of more than a few milliliters preclude the use of media constituted from natural substrates such as saliva. In this short paper, we will focus on biofilm studies, carried out in Zürich, that illustrate a few applications of one particular model with direct or indirect impact on prophylactic dentistry. The following aspects will be covered: description of the model; spatial arrangement and associative behavior of various species in biofilms; mass transport in biofilms; the biofilm model as a reliable tool to predict the in vivo efficacy of antimicrobials, and de- and remineralization of enamel exposed to biofilms in vitro.

\section{The Zürich Biofilm Model}

In contrast to most other biofilm models, our multispecies model is based on a batch culture approach and not on a continuous flow culture system. The following microorganisms representative for supragingival plaque are used to generate biofilms: Streptococcus oralis, Streptococcus sobrinus, Actinomyces naeslundii, Veillonella dispar, Fusobacterium nucleatum and Candida albicans. Biofilms are formed in 24-well cell culture dishes incubated anaerobically at $37^{\circ} \mathrm{C}$. A detailed description of the experimental procedures as well as data validating the 
Fig. 1. Schematic presentation of experimental procedures used for biofilm formation. The hydroxyapatite disks were incubated in a mixture of saliva and medium either containing 50\% saliva and 50\% medium or $70 \%$ saliva and $30 \%$ medium according to the aim of the experiment.

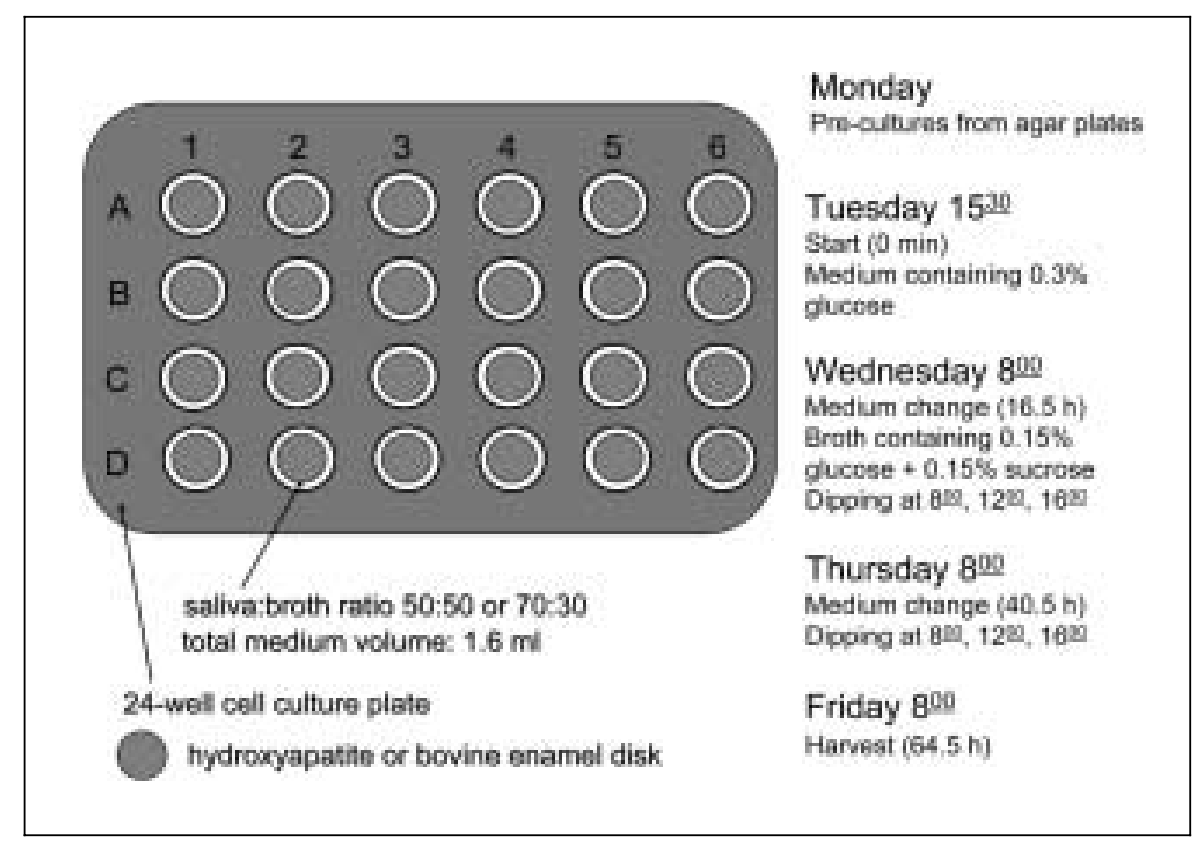

model have been published previously [Guggenheim et al., 2001a; Shapiro et al., 2002]. Therefore, only the main features are recapitulated. Biofilms are formed either on hydroxyapatite or bovine enamel disks that have been preconditioned in pooled, unstimulated saliva. An experiment, including the preparatory phase, lasts from Monday to Friday; the most important steps comprising the timing are summarized in figure 1 . In flow models or constant depth film fermenters as well as in vivo, biofilms are subjected to shear forces that are absent in a batch culture system. The disks are, therefore, dipped in saline 3 times daily (fig. 1). At each time point, the biofilms are dipped 3 times in saline, thereby being subjected to passages through an air-liquid interface. The shear forces exerted by this procedure are high and have been estimated to be $0.1 \mu \mathrm{N} /$ cell and passage [Bos et al., 1999]. When the effects of antimicrobials were investigated, the biofilms were exposed beforehand to test solutions for $1 \mathrm{~min}$, involving even a fourth passage.

\section{Spatial Arrangement and Associative Behavior of Species in Biofilms}

Polyspecies microbial consortia typically consist of cells in microcolonies embedded in exopolymer matrices. These were hitherto thought to be interwoven by a chan- nel system [Davey and O'Toole, 2000; Donlan and Costerton, 2002]. Dental plaque is a clinically relevant example of such a consortium that may mediate oral diseases. The resistance or resilience of biofilms to antimicrobials [Reid, 1999], their diffusion properties [Dibdin and Shellis, 1988; Hojo et al., 1976] and metabolic interactions between members of the consortium [Møller et al., 1998] may be linked to their distinctive architectures. In addition, roles of specific adherence mechanisms and of coadherence [Kolenbrander et al., 2000] in primary and in secondary colonization of bacteria on surfaces are widely accepted. Since these paradigms have evolved mainly from in vitro experiments with resting cell suspensions, we have subjected them to the scrutiny of experiments with growing cells in polyspecies biofilms [Guggenheim et al., 2001b].

Species-specific fluorescence-labeled antibodies in conjunction with confocal laser scanning microscopy (CLSM) allowed characterization of the spatial arrangement and interspecies associations of all members of the consortium during biofilm formation in the 50:50 model (fig. 1). In parallel, after $15 \mathrm{~min}, 16.5,40.5$ and $64.5 \mathrm{~h}$, the adherent biofilms were quantitatively analyzed using culture techniques. All species pairings were visualized in biofilms after labeling the bacteria with monoclonal antibodies coupled with 1 of 3 different fluorescent dyes in 10 nonredundant pairwise combinations. Cell number esti- 
mates by image analysis were close to culture data. Interspecies coaggregations of all strains using planktonic cells were tested in buffer and in the biofilm medium in nonredundant pairwise combinations. With the exception of $F$. nucleatum and $S$. sobrinus that coaggregated in medium as well as in buffer, the coaggregation patterns were different in the two fluids. When bacteria scraped from hydroxyapatite disks after an adherence phase of $15 \mathrm{~min}$ were analyzed by culture and image analysis, it was evident that early ( $S$. oralis, $A$. naeslundii, $V$. dispar) as well as late colonizers ( $S$. sobrinus, $F$. nucleatum) adhered in high numbers on the pellicle-covered surface (fig. 2). Image analyses revealed further that interbacterial coadherence was not a dominant mode of (indirect) adhesion to the salivary pellicle during this initial phase, since the proportion of interspecies coadhering cells was for all nonredundant pairwise combinations $<4 \%$. These findings allowed us to question the widely accepted 'Kolenbrander paradigm' proposing that specific adherence mechanisms and in vitro 'coaggregations' reflect crucial mechanisms explaining the order of colonization of bacteria in plaque [Kolenbrander and London, 1993]. We gained insight into the structural features of all species during biofilm development, and into the associative behavior of the strains within the biofilm, that were classified into 5 spatial types; some examples are shown in figure 3. More information is provided by Guggenheim et al. [2001b].

\section{Mass Transport of Macromolecules within an in vitro Grown Biofilm}

The ability to generate biofilms with high repeatability and the technical skills to visualize these in high quality in native form by CLSM allowed us to tackle experimentally their diffusion properties. Knowledge of the kinetics of mass transport within oral biofilms is essential for understanding how they achieve their characteristic architecture, how they manifest their pathogenic potential and for optimizing strategies to control or eradicate biofilms.

64.5-hour biofilms of the 70:30 model (fig. 1), formed on hydroxyapatite disks preconditioned with saliva, were incubated for defined periods at room temperature with fluorescent markers with molecular weights ranging from 3 to $900 \mathrm{kD}$. Dextrans (3, 10, 40, $70 \mathrm{kD}), \operatorname{IgG}(150 \mathrm{kD})$, $\mathrm{F}\left(\mathrm{ab}^{\prime}\right)_{2}$ fragments of $\operatorname{IgG}(100 \mathrm{kD})$, R-phycoerythrin $(240 \mathrm{kD})$ and IgM $(900 \mathrm{kD})$ amongst others were used in recently published experiments [Thurnheer et al., 2003]. Biofilm-carrying disks were incubated with the fluores-

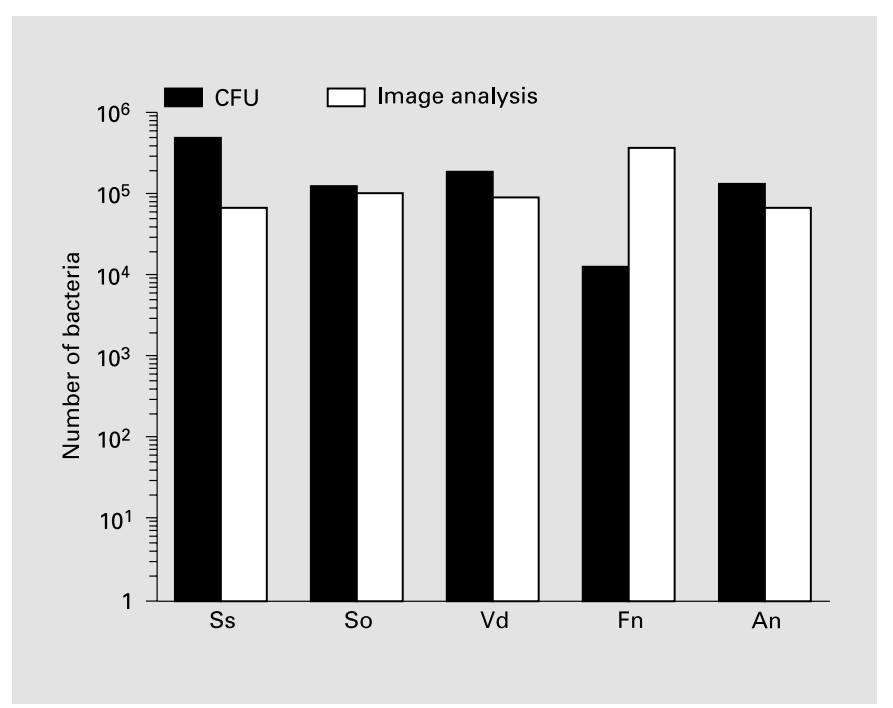

Fig. 2. Number of bacteria as estimated by culture (CFU) or image analysis on salivary-coated hydroxyapatite disks after an initial adherence phase of 15 min: S. sobrinus (Ss), S. oralis (So), V. dispar $(\mathrm{Vd})$, F. nucleatum $(\mathrm{Fn})$, A. naeslundii (An).

cent probes for $2,60,120,300$ or $600 \mathrm{~s}$, washed by two 60 -second dips in saline and then embedded upside down in Mowiol [Guggenheim et al., 2001b] to block further diffusion. They were examined by CLSM at 5 randomly selected positions. A few examples of the images collected are shown in figure 4 . It is evident that $240-\mathrm{kD}$ phycoerythrin penetrated poorly into biofilms, whereas $900-\mathrm{kD}$ IgM accumulated on the surface and penetrated hardly at all. From reported hydrodynamic radii for these molecules, the limiting diameter of the biofilm pores can be estimated as slightly greater than $11 \mathrm{~nm}$. A control experiment showed that microspheres $(\varnothing=20 \mathrm{~nm})$ did not penetrate the biofilm either. Subsequent analyses revealed that the mean square penetration depth for all tested macromolecules except IgM and 3-kD dextran increased linearly with time, diffusion coefficients being linearly proportional to the cube root of the molecular weight of the probes. Diffusion in the biofilms was markedly slower than in water. Analysis of diffusion phenomena through oral biofilms suggested tortuosity as the most probable explanation. The retardation of molecules with hydrodynamic radii < approx. $10 \mathrm{~nm}$ and the generally proposed existence of a channel network in biofilms [Hall-Stoodley and Stoodley, 2002; Wood et al., 2000] with diameters in the micrometer range are conflicting notions. Such 'black holes' are also present in Syto13 or triple fluorescent in 

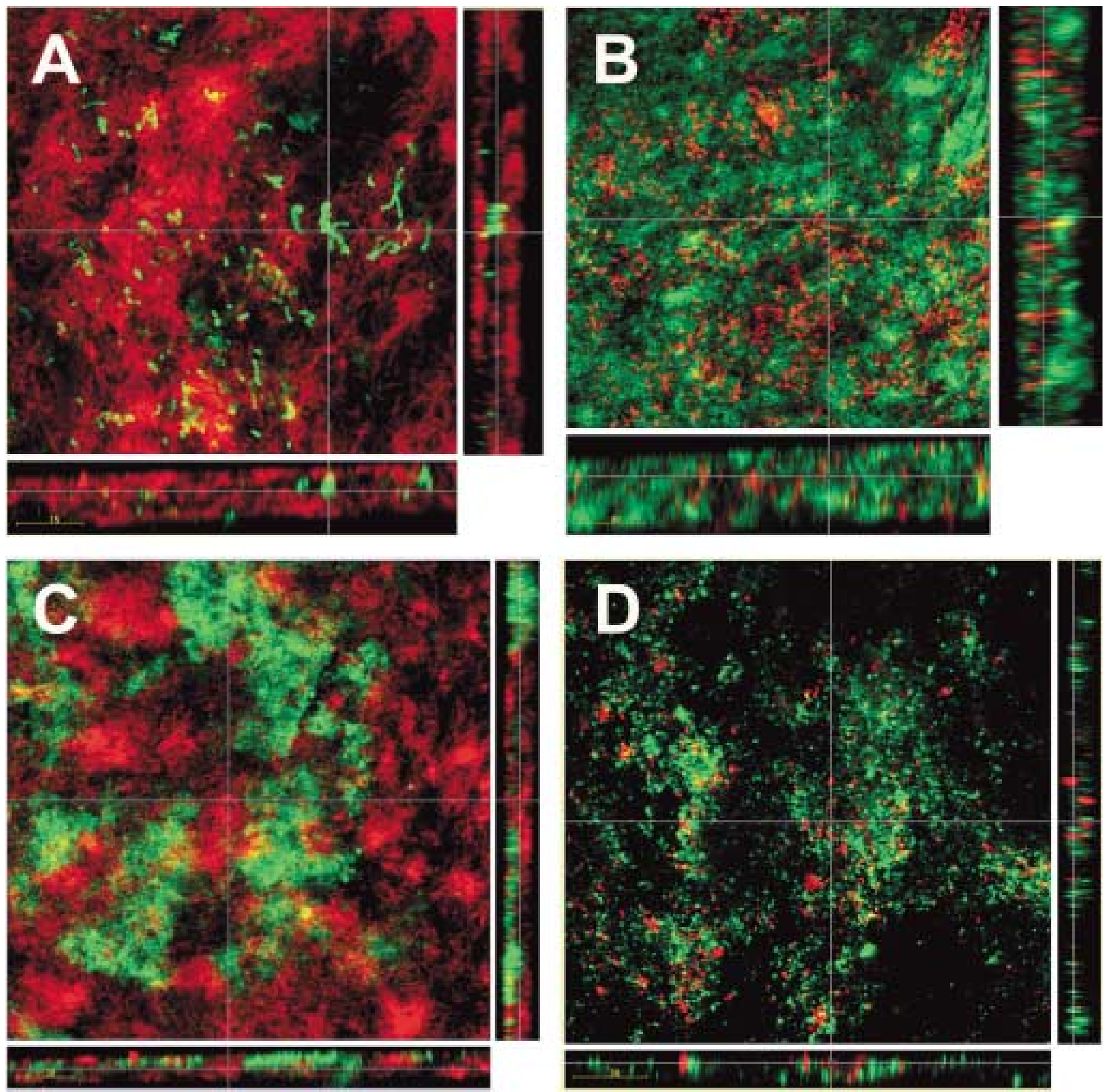

Fig. 3. CLSM images of 64.5-hour biofilms (50:50 model) stained with species-specific monoclonal antibodies. A $S$. sobrinus (green) plus $F$. nucleatum (red). B $F$. nucleatum (green) plus A. naeslundii (red). C $S$. oralis (green) plus F. nucleatum (red). D V. dispar (green) plus $S$. sobrinus (red).

situ hybridization [Thurnheer et al., 2004] stained biofilms (fig. 5A, B). However, when such biofilms are in addition stained with the exopolysaccharide stain Calcofluor [Thurnheer et al., 2003], it becomes evident that multispecies biofilms formed in the presence of oral strep- tococci and sucrose consist of microbial microcolonies embedded in a compact polysaccharide hydrogel without channels (fig. 5C). Diffusion experiments with such double-stained biofilms revealed that dextrans $\geq 10 \mathrm{kD}$ cannot diffuse through the extracellular polysaccharide moi- 

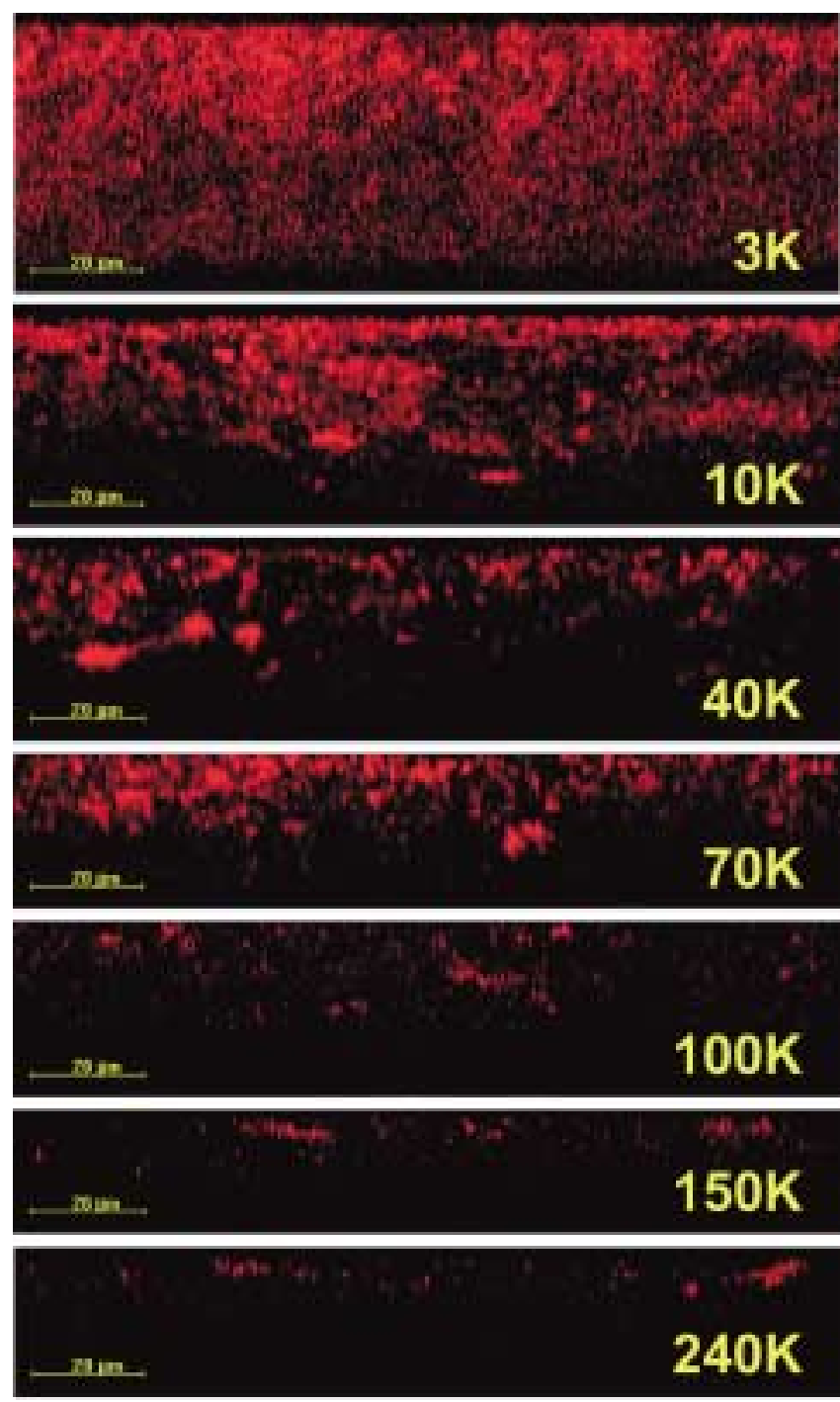

Fig. 4. CLSM images of cross-sections of 64.5-hour biofilms (70:30 model) showing the diffusion of macromolecules with different molecular weights after $120 \mathrm{~s} \mathrm{(3-kD} \mathrm{dextran)}$ or after $600 \mathrm{~s}$ (10- to 240$\mathrm{kD}$ probes).

ety and must find their way on winding pathways through microcolonies, thus providing a more direct picture of the term tortuosity (fig. 5D). In contrast, 3-kD dextrans are able to diffuse through the exopolysaccharide moiety having a pore diameter of $2.8-4.6 \mathrm{~nm}$ (data not shown), thus explaining the higher diffusion rate and the lower tortuosity. These findings may explain the lower cariogenic potential of starch in comparison to low-molecular-weight saccharides.

Impact of Biofilm Models on Dentistry

\section{The Zürich Biofilm Model as Reliable Predictor for the Clinical Efficacy of Antimicrobials}

For decades the potential of antimicrobials for oral use was tested in classical MIC and MBC tests utilizing planktonic monocultures and prolonged contact times. In comparison to clinical tests the resulting inhibitory concentrations were 100-1,000 times too low [Shapiro and Guggenheim, 1998]. Thus, they allowed only relative comparisons and were poorly predictive for the clinical efficacy of antiseptic mouth rinses. The obvious reasons are the brief exposure times to e.g. mouth rinses $(<3 \mathrm{~min} /$ day $)$ and proliferation of surviving microorganisms in plaque during the rest of the day and night between rinsings. It is furthermore widely accepted that bacteria in biofilms express a more resistant phenotype than planktonic bacteria [Marsh, 2003]. Using the 50:50 biofilm model (fig. 1), we devised a simple in vitro model of supragingival plaque whose response towards triclosan and chlorhexidine digluconate (CHX) mimicked closely clinical results reported for these antimicrobial agents [Guggenheim et al., 2001a]. However, when biofilms were exposed 3 times daily during $1 \mathrm{~min}$ to commercially available mouth rinses, in particular in low concentrations, the effect did not perfectly match results of clinical studies. Therefore, efforts have been directed towards fine-tuning the model in order to further improve the correspondence between the biofilm response to antimicrobial agents and the effect of these agents on plaque in vivo. It appeared that the effect of antimicrobials on the biofilm was dependent on its growth rate, which is in turn related to the ratio of saliva and medium in the incubation fluid. Using $0.1,0.12$ and $0.2 \%$ CHX and a saliva:medium ratio of $70: 30$, a dose-dependent response was observed, and this ratio was adopted for subsequent experiments (' $70: 30$ model'). The discriminative power of the model is illustrated in figure 6.

The efficacies of 12 different mouth rinses - proprietary products containing $\mathrm{CHX}$, hexetidine, octenidine, triclosan, plant extracts or amine fluoride/stannous fluoride - vis-à-vis biofilm inhibition were compared by Shapiro et al. [2002]. An excerpt from these data is shown in figure 7. In general, there is a good overall agreement between results obtained using our in vitro model and those reported in relevant clinical trials. However, there is a discrepancy in antimicrobial efficiency between pure CHX-containing solutions and some commercial CHX mouth rinses due to the vitiating effect of product formulation [Shapiro et al., 2002]. On the other hand, auxiliary ingredients may also increase the efficacy of a pure com-

Caries Res 2004;38:212-222 

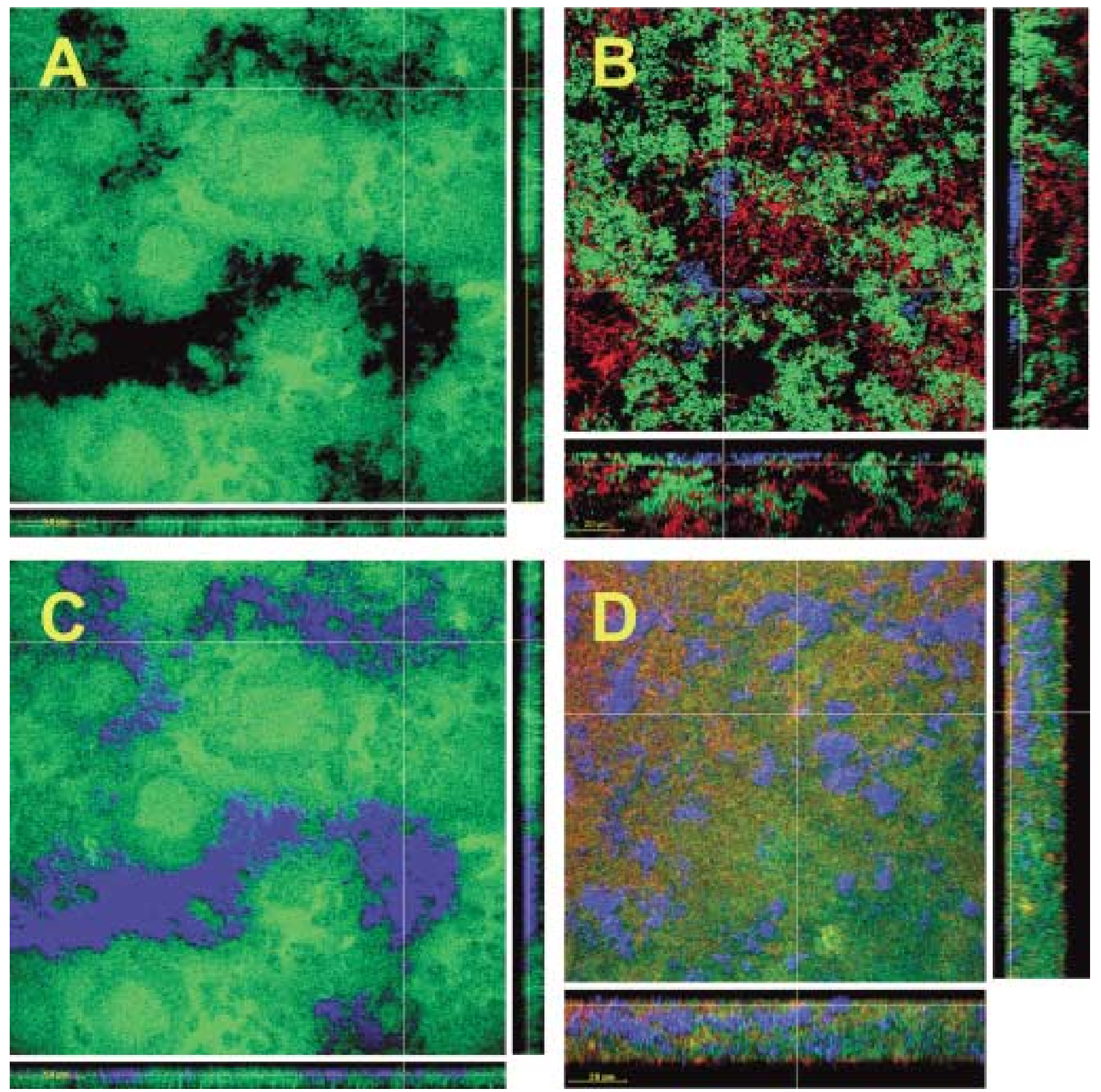

Fig. 5. CLSM images of 6-species biofilms stained for all bacteria with Syto13 (A), for F. nucleatum (red), S. oralis (green) and $V$. dispar (blue) by triple fluorescent in situ hybridization (B), for bacteria (Syto13) and exopolysaccharides (Calcofluor, blue) (C) and for bacteria (Syto13, green), 10-kD dextran (red) and exopolysaccharides (Calcofluor, blue) (D). The images $\mathbf{A}$ and $\mathbf{C}$ show the same spot of the biofilm. 
Fig. 6. Box plots showing the effect of 2 concentrations $(0.1$ and $0.2 \%$, i.e. 1.1 and $2.2 \mathrm{mM})$ of CHX and $0.2 \%(6.9 \mathrm{~m} M)$ triclosan (TC) on 5-species biofilm formation compared to a water control $(n=9)$. Differences between control and all treatments were highly significant at the $99.9 \%$ level. Differences between treatments were not significant.

Fig. 7. Box plots depicting viable cell recovery from 6-species biofilms $(n=9)$ treated with different commercially available mouth rinses. Significant differences between treatments at the 99 or $95 \%$ level are indicated by $* *$ and $*$, respectively; n.s. $=$ not significant.
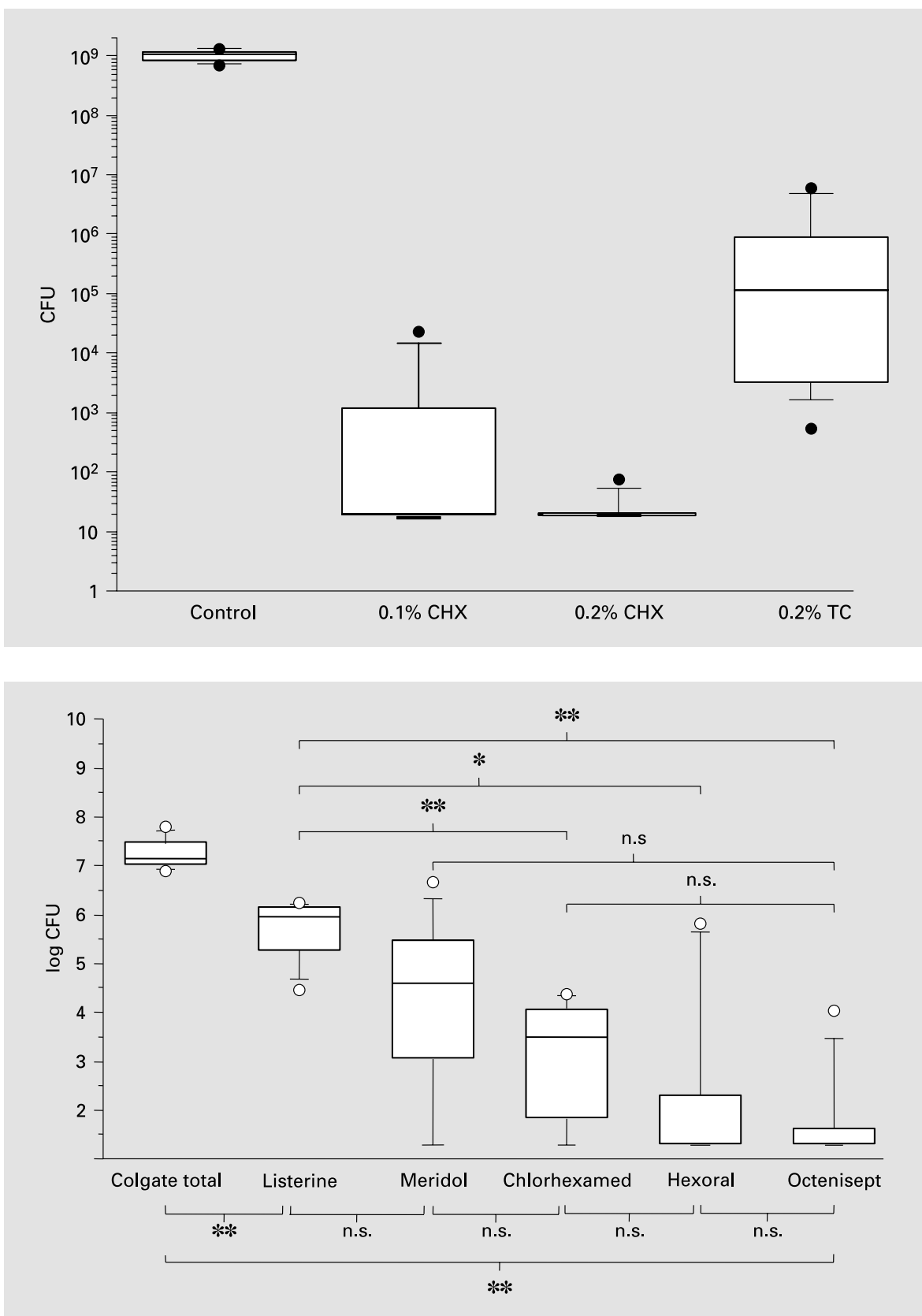

pound [Shapiro et al., 2002]. When comparing the effect of antimicrobials, the time point of analysis after the last exposure is crucial. An ideal product should prevent plaque regrowth over extended periods and we chose $16 \mathrm{~h}$. If an antimicrobial has a low microbicidal effect, even slight differences in the numbers of survivors can lead to large variation due to biofilm growth in the posttreatment period. Although our in vitro model cannot mirror oral distribution of a mouth rinse, it has the great advantage that a mouth rinse can be applied to and removed from the system virtually instantaneously, simulating the brief exposure of supragingival plaque to mouth rinses. In addition, the model can be set up in any microbiology laboratory with standard equipment. It is at present the only model allowing in vitro a reliable prognosis of the in vivo performance of antimicrobials for oral use and is thus a valuable tool for preclinical evaluation of antiplaque formulations. 

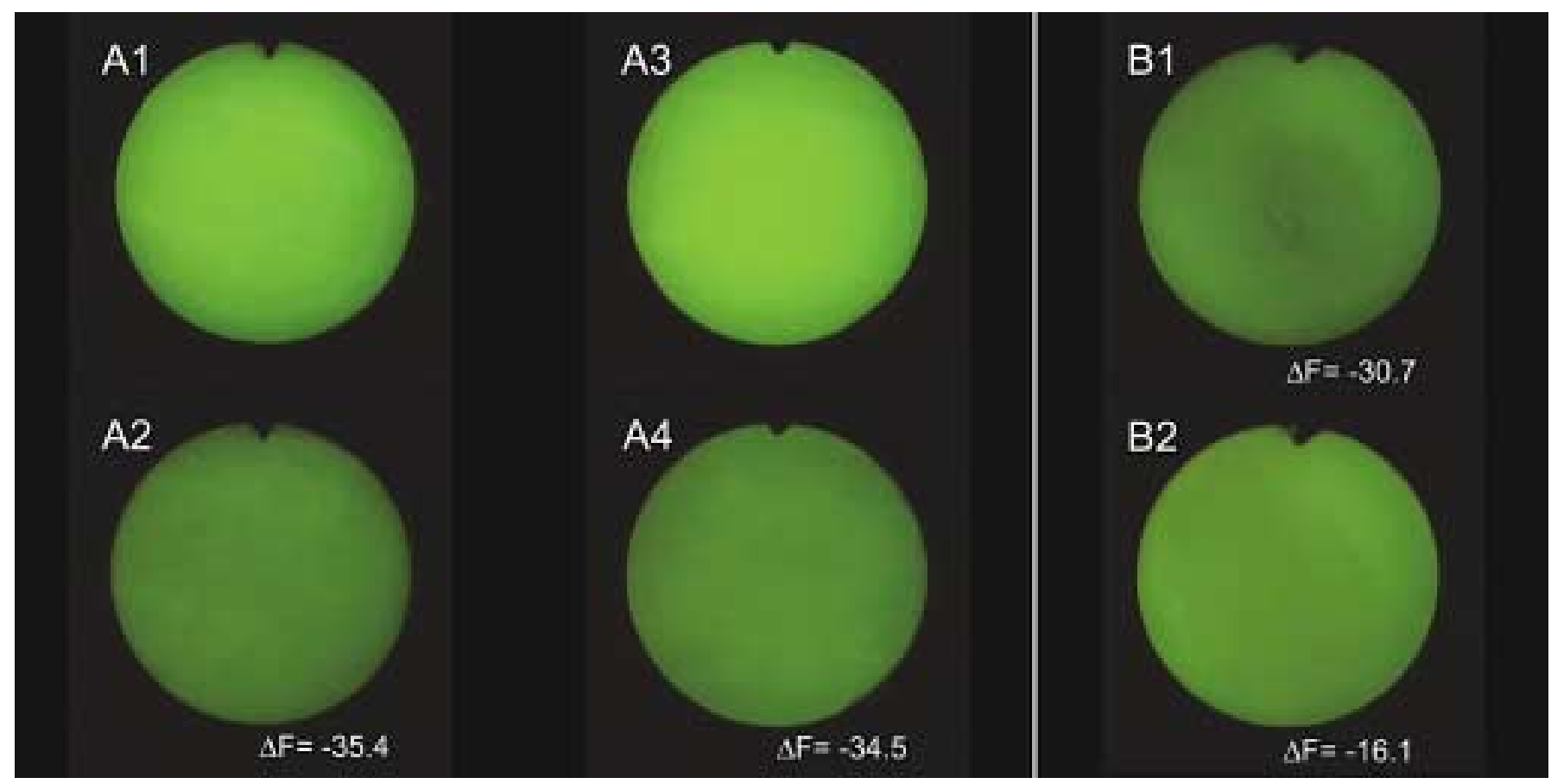

Fig. 8. QLF images of bovine enamel disks before $(\mathbf{A 1}, \mathbf{A 3})$ and after demineralization (A2, A4). B1 Image of a disk demineralized with an acidic gel. B2 The same disk after pretreatment with Elmex gel diluted 1:5 and remineraliza-

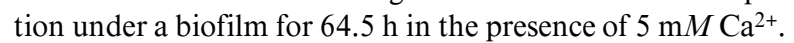

\section{Demineralization and Remineralization of Enamel in the Zürich Biofilm Model}

More recently, we explored whether our biofilm model could be used to achieve demineralization and remineralization of bovine enamel in vitro [Guggenheim et al., 2003]. The necessary prerequisite was to find a method allowing a reproducible and rapid assessment of the degree of mineralization of enamel. Bovine enamel disks were prepared from incisors of cows. Demineralization was measured by quantitative light-induced fluorescence (QLF) [Al-Khateeb et al., 1997; van der Veen and de Josselin de Jong, 2000] using a modification of the 'in vivo' technique, with prototype hardware and software. We found buffer and carbohydrate concentrations in the medium to be the main parameters controlling demineralization. These variables were tested in a checkerboard arrangement in order to find conditions resulting in substantial demineralization in the 70:30 biofilm model.

Six buffer concentrations from 0 to $100 \% 0.66 \mathrm{M}$ Sørensen phosphate buffer, $\mathrm{pH} 7.2$, were applied in the medium. In the vertical direction, 4 carbohydrate concentrations (glucose + sucrose $1: 1$ ) between 0.5 and $5 \%$ were tested. At a concentration of $0.5 \%$ carbohydrate, no demineralizations were observed at any buffer concentration. At a concentration of $1 \%$ carbohydrate, demineralizations were observed that decreased with increasing buffer concentration. At a concentration $>1 \%$ carbohydrate bovine enamel was strongly demineralized independently of the buffer strength. The results led to the choice of $0 \%$ buffer and $1 \%$ carbohydrate, which gave a mean demineralization of $-30.5 \pm 3.3 \%(n=6)$ with a very narrow range (-27.3 to $-35.4 \%)$. In figure $8 \mathrm{~A}$, representative QLF images of disks before and after demineralization are shown. Differences in fluorescence before and after biofilm exposure are clearly visible.

For remineralization experiments, enamel disks demineralized in vitro by acid gels [Schmidlin et al., 2002] were used. The degree of demineralization was assessed by QLF prior to using them as substrate for biofilm formation in the 70:30 model. Ideally, disks with a $\Delta \mathrm{F}$ score of -25 to $-30 \%$ were chosen. We found fluoride and calcium to cause heavy precipitation in the 70:30 biofilm medium. Therefore, fluoride was applied prior to pellicle formation. Enamel disks were brushed for 2 min with fluoride preparations, stored in a wet chamber for $2 \mathrm{~h}$ and 


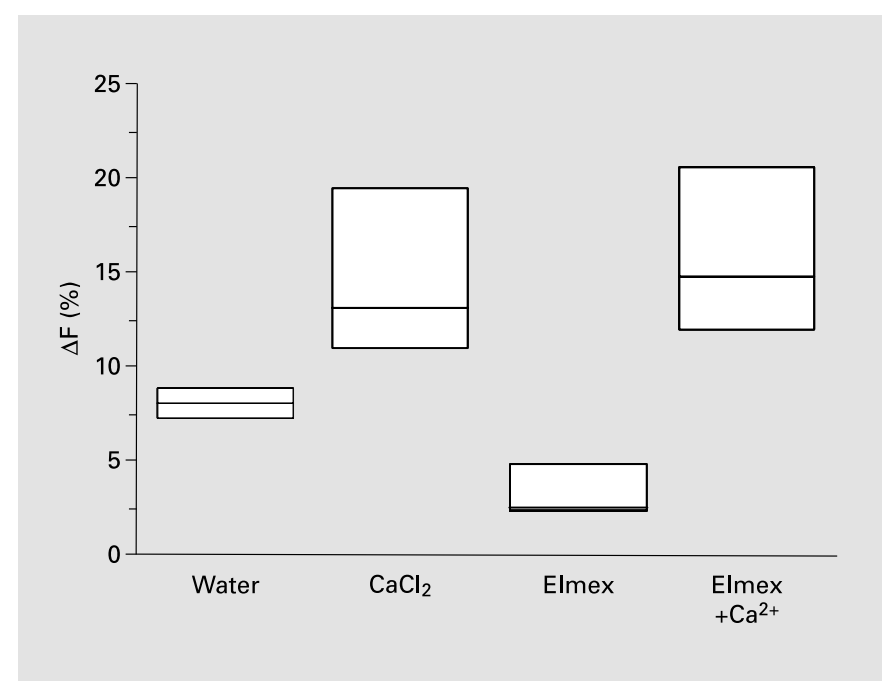

Fig. 9. Remineralization of bovine enamel disks under biofilms is expressed as increase in $\Delta \mathrm{F}$. A water control is compared with disks incubated with an addition of $5 \mathrm{mM \textrm {Ca } ^ { 2 + }}$ to the medium. Disks pretreated with a 1:5 diluted Elmex gel only were compared with disks after fluoride pretreatment and additional incubation with $5 \mathrm{mM}$ $\mathrm{Ca}^{2+}$ in the medium.

subsequently rinsed for $15 \mathrm{~s}$ with tap water. Biofilms were formed on fluoride or water-pretreated enamel disks exposed to $5 \mathrm{~m} M$ calcium in the medium for $64.5 \mathrm{~h} . \Delta \mathrm{F}$ was calculated before and after exposure to biofilms. Results shown in figure 9 reveal that the addition of $5 \mathrm{mM}$ calcium chloride resulted in a remineralization of approximately $7.5 \%$. Fluoride-pretreated disks exposed in addition to $5 \mathrm{~m} M$ calcium remineralized by $12.5 \%$. Remineralization was comparatively slow but could still be seen by eye (fig. 8B). Thus, our model may be used for studying demineralization and remineralization under biofilms. In particular, inhibition of demineralization, the effect of different microbiological ecologies, the pathogenicity of single strains and the effect of different fluoride pretreatments on enamel remineralization may be studied.

\section{Concluding Remarks}

The Zürich in vitro biofilm model is reproducible and reliable. It may be used for the study of basic, but also for very application-oriented questions that could not be addressed before. Only very few applications could be shown because of space limits; there would have been many others, and there is even much more to explore. The use of biofilm models allows us to address a multitude of questions that could hitherto not be studied with planktonic monocultures. A new area of research in oral biology is now open with promising prospects for preventive dentistry.

We have questioned paradigms that have evolved by extrapolating characteristics from mainly Pseudomonas aeruginosa biofilms to oral biofilms or that have been derived from results of in vitro studies with planktonic bacteria under nonphysiological conditions. It is now more and more accepted that in different environments biofilms with widely different properties are formed and that the prevailing growth conditions are the overall dominating factor [Klausen et al., 2003].

\section{Acknowledgments}

The authors are grateful for excellent technical assistance by André Meier, Martin Gander and Verena Osterwalder. The work was supported by the Universities of Zürich and Bergen, Patentmedelsfonden för Odontologisk Profylaxforskning (Sweden), Colgates Forskningsfond (Norway) and A/S Norsk Dental Depots Fond for Odontologisk Forskning (Bergen).

\section{References}

Al-Khateeb S, Oliveby A, de Josselin de Jong E, Angmar Månsson B: Laser fluorescence quantification of remineralisation in situ of incipient enamel lesions: Influence of fluoride supplements. Caries Res 1997;31:132-140.

Bos R, van der Mei HC, Busscher HJ: Physicochemistry of initial microbial adhesive interactions - Its mechanisms and methods for study. FEMS Microbiol Rev 1999;23:179-230.

Bowden GH: Controlled environment model for accumulation of biofilms of oral bacteria. Methods Enzymol 1999;310:216-224.
Bradshaw DJ, Marsh PD, Schilling KM, Cummins D: A modified chemostat system to study the ecology of oral biofilms. J Appl Bacteriol 1996; 80:124-130.

Carlsson J: Dental plaque as a source of salivary streptococci. Odont Rev 1967;18:173-178.

Christersson CE, Fornalik MS, Baier RE, Glantz P-O: In vitro attachment of oral microorganisms to solid surfaces: Evaluation of a controlled flow method. Scand J Dent Res 1987; 95:151-158.

Costerton JW, Lewandowski Z, Caldwell DE, Korber DR, Lappin-Scott HM: Microbial biofilms. Annu Rev Microbiol 1995;49:711-745.
Costerton JW, Lewandowski Z, de Beer D, Caldwell D, Korber D, James G: Biofilms, the customized microniche. J Bacteriol 1994;176: 2137-2142.

Davey ME, O'Toole GA: Microbial biofilms: From ecology to molecular genetics. Microbiol Mol Biol Rev 2000;64:847-867.

Davies DG, Parsek MR, Pearson JP, Iglewski BH, Costerton JW, Greenberg EP: The involvement of cell-to-cell signals in the development of a bacterial biofilm. Science 1998;280:295298. 
Dibdin GH, Shellis RP: Physical and biochemical studies of Streptococcus mutans sediments suggest new factors linking the cariogenicity of plaque with its extracellular polysaccharide content. J Dent Res 1988;67:890-895.

Donlan RM, Costerton JW: Biofilms: Survival mechanisms of clinically relevant microorganisms. Clin Microbiol Rev 2002;15:167-193.

Gibbons RJ: Bacteriology of dental caries. J Dent Res 1964;43:1021-1028.

Gilbert P, Das J, Foley I: Biofilm susceptibility to antimicrobials. Adv Dent Res 1997;11:160167.

Guggenheim B: Streptococci of dental plaques. Caries Res 1968;2:147-163.

Guggenheim B: Extracellular polysaccharides and microbial plaque. Int Dent J 1970;20:657678.

Guggenheim B, Giertsen E, Gmür R: Demineralization and remineralization of enamel in the Zürich biofilm model. J Dent Res 2003;82: 1861.

Guggenheim B, Giertsen E, Schüpbach P, Shapiro $\mathrm{S}$ : Validation of an in vitro biofilm model of supragingival plaque. J Dent Res 2001a;80: 363-370.

Guggenheim B, König KG, Mühlemann HR: Modifications of the oral bacterial flora and their influence on dental caries in the rat. I. The effects of inoculating 4 labelled strains of streptococci. Helv Odont Acta 1965;9:121-129.

Guggenheim M, Shapiro S, Gmür R, Guggenheim B: Spatial arrangements and associative behavior of species in an in vitro oral biofilm model. Appl Environ Microbiol 2001b;67:1343-1350.

Hall-Stoodley L, Stoodley P: Developmental regulation of microbial biofilms. Curr Opin Biotechnol 2002;13:228-233.

Hardie JM, Thomson PL, South RJ, Marsh PD, Bowden GH, McKee AS, Fillery ED, Slack GL: A longitudinal epidemiological study on denta plaque and the development of dental caries Interim results after two years. J Dent Res 1977;56(special issue):C90-C98.

Herles S, Olsen S, Afflitto J, Gaffar A: Chemostat flow cell system: An in vitro model for the evaluation of antiplaque agents. J Dent Res 1994; 73:1748-1755.
Hojo S, Higuchi M, Araya S: Glucan inhibition of diffusion in plaque. J Dent Res 1976;55:169.

van Houte J: Role of micro-organisms in caries etiology. J Dent Res 1994;73:672-681.

Keyes PH: The infectious and transmissible nature of experimental dental caries: Findings and implications. Arch Oral Biol 1960;1:304-320.

Kinniment SL, Wimpenny JWI, Adams D, Marsh PD: Development of a steady-state oral microbial biofilm community using the constantdepth film fermenter. Microbiology 1996;142: 631-638.

Klausen M, Heydorn A, Ragas P, Lambertsen L, Aaes-Jørgensen A, Molin S, Tolker-Nielsen T: Biofilm formation by Pseudomonas aeruginosa wild type, flagella and type IV pili mutants. Mol Microbiol 2003;48:1511-1524.

Kolenbrander PE, Andersen RN, Kazmerak KM: Coaggregation and coadhesion in oral biofilms; in Allison DG, Gilbert P, Lappin-Scott HM (eds): Community Structure and Co-Operation in Biofilms. Cambridge, Cambridge University Press, 2000, pp 65-85.

Kolenbrander PE, London J: Adhere today, here tomorrow: Oral bacterial adherence. J Bacteriol 1993; 175:3247-3252.

Kristoffersson K, Gröndahl H-G, Bratthall D: The more Streptococcus mutans, the more caries on approximal surfaces. J Dent Res 1985;64:5861.

Larsen T, Fiehn NE: Development of a flow method for susceptibility testing of oral biofilms in vitro. APMIS 1995;103:339-344.

Liljemark WF, Bloomquist C: Human oral microbial ecology and dental caries and periodontal diseases. Crit Rev Oral Biol Med 1996;7:180198.

Loesche WJ: Role of Streptococcus mutans in human dental decay. Microb Rev 1986;50:353380 .

Marsh PD: Microbial ecology of dental plaque and its significance in health and disease. Adv Dent Res 1994;8:63-71.

Marsh PD: Plaque as a biofilm: Pharmacological principles of drug delivery and action in the sub- and supragingival environment. Oral Dis 2003;9(suppl 1):16-22.

Miller WD: The Micro-Organisms of the Human Mouth: The Local and General Diseases Which Are Caused by Them. Unaltered reprint of the original work by Willoughby D. Miller (18531907) published in 1890 in Philadelphia. Basel, Karger, 1973.
Møller S, Sternberg C, Andersen JB, Christensen BB, Ramos JL, Givskov M, Molin S: In situ gene expression in mixed-culture biofilms: Evidence of metabolic interactions between community members. Appl Environ Microbiol 1998;64:721-732.

Ooshima T, Matsumura M, Hoshino T, Kawabata S, Sobue S, Fujiwara T: Contributions of three glucosyltransferases to sucrose-dependent adherence of Streptococcus mutans. J Dent Res 2001;80:1672-1677.

Reid G: Biofilms in infectious disease and on medical devices. Int J Antimicrob Agents 1999;11: 223-226.

Schmidlin PR, Tepper SA, Scriba H, Lutz F: In vitro assessment of incipient approximal carious lesions using computer-assisted densitometric image analysis. J Dent 2002;30:305311

Shapiro S, Giertsen E, Guggenheim B: An in vitro oral biofilm model for comparing the efficacy of antimicrobial mouth rinses. Caries Res 2002;36:93-99.

Shapiro S, Guggenheim B: Chemoprophylaxis in the oral cavity: 'plus on change les choses, plus elles devraient rester les mêmes'; in Guggenheim B, Shapiro S (eds): Oral Biology at the Turn of the Century: Misconceptions, Truths, Challenges and Prospects. Basel, Karger, 1998, pp 226-238.

Sjollema J, Busscher HJ, Weerkamp AH: Real-time enumeration of adhering microorganisms in a parallel plate flow cell using automated image analysis. J Microbiol Methods 1989;9:73-78.

Thurnheer T, Gmür R, Guggenheim B: Multiplex FISH analysis of a six-species bacterial biofilm. J Microbiol Methods 2004;56:37-47.

Thurnheer T, Gmür R, Shapiro S, Guggenheim B: Mass transport of macromolecules within an in vitro model of supragingival plaque. Appl Environ Microbiol 2003;69:1702-1709.

van der Veen MH, de Josselin de Jong E: Application of quantitative light-induced fluorescence for assessing early caries lesions. Monogr Oral Sci 2000; 17:144-162.

Wood SR, Kirkham J, Marsh PD, Shore RC, Nattress B, Robinson C: Architecture of intact natural human plaque biofilms studied by confocal laser scanning microscopy. J Dent Res 2000;79:21-27. 